\title{
Control parameters for retinex
}

\author{
Brian Funt ${ }^{*}$ and Florian Ciurea ${ }^{*}$ \\ School of Computing Science, Simon Fraser University
}

\begin{abstract}
The recently published Matlab implementation of the retinex algorithm ${ }^{2}$ has free parameters for the user to specify. The parameters include the number of iterations to perform at each spatial scale, the viewing angle, image resolution, and the lookup table function (post-lut) to be applied upon completion of the main retinex computation. These parameters were specifically left unspecified in ${ }^{2}$ since the previous descriptions of retinex upon which the new Matlab implementations were based do not define them. In this paper we determine values for these parameters based on a best fit to the experimental data provided by McCann et. al. ${ }^{9}$
\end{abstract}

Keywords: Retinex, color vision, human vision, color constancy.

\section{INTRODUCTION}

The retinex model for the computation of lightness was introduced by Land and McCann ${ }^{7}$ in 1971. Land and his colleagues later described additional improvements to the original method ${ }^{9,5,1,6,8}$. These further refinements were mainly designed to improve computational efficiency while preserving the retinex principle of comparing pixel values from different spatial locations. Matlab code for two of the main retinex algorithms is provided in ${ }^{2}$ by Funt et. al.

Even though the retinex algorithm is well documented, there are still many things which need to be specified before it can be used as a model of human color or lightness perception. In particular, there are parameters which are sensitive to both the spatial frequency and the dynamic range of the input image. We estimate values for these parameters based on fitting the experimental data obtained by McCann, McKee and Taylor. ${ }^{9}$ We will refer to their study as the MMT (McCann-McKee-Taylor) experiment. A central part of the MMT experiment involves haploscopic matching of Munsell papers arranged in a Mondrian display ${ }^{9}$. Our procedure is to reconstruct digital images corresponding to the cone responses of the standard observer, and then run the retinex algorithm on that data while varying the algorithm's parameters to find those for which the program's output best matches the MMT corresponding color data.

\section{PREPARING RETINEX INPUT DATA}

Our goal is to determine the values of the free retinex parameters which make retinex work as accurately as possible as a model of color appearance in complex visual scenes. In the MMT experiment ${ }^{9}$, subjects alternately viewed a Mondrian with one eye and a Munsell chip with the other eye. For each colored area in the Mondrian, the subjects chose a matching Munsell chip. The experiment was repeated under 5 different combinations of three narrowband illuminants. The results in ${ }^{9}$ are reported in terms of the designators of the matching Munsell chips. In this paper we rely on the corresponding CIE tristimulus values estimated by Nayatani et. al. ${ }^{10}$.

Our first step was to construct an LMS image of the Mondrian used in the MMT experiment as it would be under each of the 5 illuminants. The layout of the color patches in the Mondrian is given in MMT ${ }^{9}$. We convert the corresponding $\mathrm{XYZ}$ of each patch as estimated by Nayatani ${ }^{10}$ to cone quanta catch values using the following transformation ${ }^{4}$ :

$$
\begin{aligned}
& \mathrm{L}=0.38971 \mathrm{X}+0.68898 \mathrm{Y}+0.07868 \mathrm{Z} \\
& \mathrm{M}=-0.22981 \mathrm{X}+1.18340 \mathrm{Y}+0.04641 \mathrm{Z} \\
& \mathrm{S}=1.00 \mathrm{Z}
\end{aligned}
$$


Similarly, the XYZs of the matching Munsell chips are converted to LMS. The natural logarithm of each L, M, S value is then taken since the Matlab retinex implementations ${ }^{2}$ require the logarithm of the image as input. Retinex is run on each of the L, M and S channels independently.

\section{POST RETINEX PROCESSING}

The post-retinex processing consists of four stages: exponentiation, scaling to white, conversion to Munsell Value scale, and compensation for differences in overall illumination intensity. Exponentiation of the retinex output simply compensates for the logarithm which was applied to the input data. Scaling to white is required because the retinex algorithm normalizes each of the LMS channels to 1 . After retinex processing an ideal white patch will result in $(1,1,1)$; however, the LMS value of the Munsell white (MMT area K) under the 'white' illumination in the MMT experiment is $(92.55,72.84,49.23)$. Hence, we scaled the retinex output values to make the retinex white equal the MMT white. The three scaling factors, one for each channel, were then held constant across the 5 MMT illumination conditions.

The second post-retinex stage is to convert to Munsell Value scale, which is required because McCann et. al. compare the colors in the Mondrian to the matching Munsell chips using it. They convert integrated reflectance $\rho$ (e.g., L/Lwhite) to Munsell Value using the approximation ${ }^{3}$ :

$$
\mathrm{V}=2.539 \rho^{1 / 3}-1.838 \text { for } \rho>0.384 \%
$$

The third stage is to compensate for differences in overall illumination intensity between the test and match conditions based on the data in Figure 8 of MMT. McCann et. al. found that overall intensity affected subjects' matches. Hence, we incorporated their correction factor as a function of the ratio of overall illumination between the two scenes. By analyzing MMT Figure 8, we computed the correction to be added to the retinex output converted to Munsell Value, based on the scene radiances $\mathrm{E}$ at $630-\mathrm{nm}, 530-\mathrm{nm}$ and $450-\mathrm{nm}$ as:

$$
\begin{aligned}
& \text { Correction }^{630}=1.53 \times \log _{10}\left(\mathrm{E}^{630}{ }_{\text {Mondrian }} / \mathrm{E}^{630}{ }_{\text {Munsell }}\right)+0.04 \\
& \text { Correction }^{530}=1.19 \times \log _{10}\left(\mathrm{E}^{530}{ }_{\text {Mondrian }} / \mathrm{E}^{530}{ }_{\text {Munsell }}\right)+0.11 \\
& \text { Correction }^{450}=0.93 \times \log _{10}\left(\mathrm{E}^{450}{ }_{\text {Mondrian }} / \mathrm{E}^{450}{ }_{\text {Munsell }}\right)+0.01
\end{aligned}
$$

\section{RESULTS}

To establish the optimum choice for the number of retinex iterations, we ran retinex with the number of iterations (parameter nIterations in the Matlab implementation ${ }^{2}$ ) varying from 1 to 500 . The post-retinex processing described above was then applied in each case. For each iteration setting, we computed the difference between the final retinex prediction and the matching Munsell chip data found in the MMT experiment. The image difference measure is the RMS over all pixels of the following single pixel difference measure:

$$
d_{3}\left(R^{c}(i, j)-M^{c}(i, j)\right)=\sqrt{\sum_{c=L, M, S}\left(R^{c}(i, j)-M^{c}(i, j)\right)^{2}}
$$

$R^{c}(i, j)$ denotes the pixel value at channel $c$ for retinex output including post-processing; $M^{c}(i, j)$ denotes the pixel value at channel $\mathrm{c}$ for an image of the Mondrian made up of Munsell matching chips.

We found that for each of the five different MMT experimental setups- "gray", "red", "blue", "green" and "yellow"different numbers of iterations were required to give the best match to the matching Munsell data. Although the number of iterations varied across the cases, 33 iterations gave the best overall result.

Image resolution is another variable which must be considered. To determine how the optimum number of iterations might be affected by image resolution, we constructed images of otherwise identical Mondrians at resolutions of $128 \times 128,256 \times 256,512 \times 512$ and $1024 \times 1024$. Contrary to what might be expected, we found almost no change in the optimum number of retinex iterations required as a function of image resolution. 
The following graphs illustrate how the number of iterations affect the distance between the retinex prediction and the actual image as seen by the observers, for each experiment. For these tests we ran retinex with input images of $256 \times 256$ pixels.

"gray" experiment

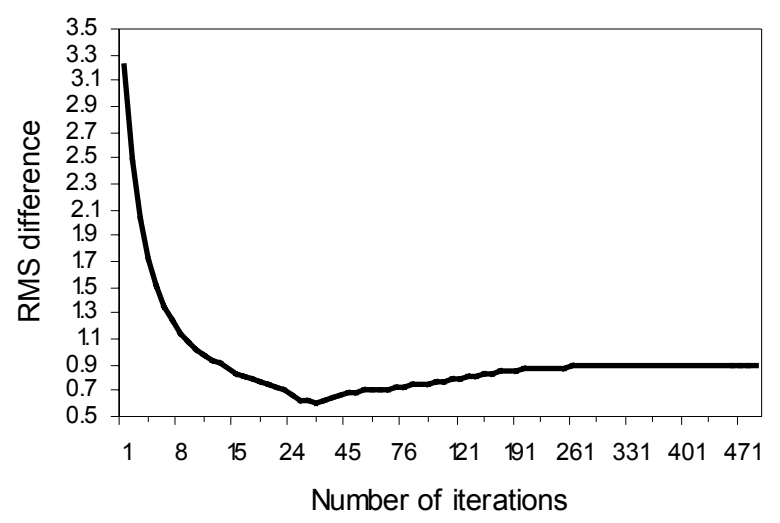

"blue" experiment

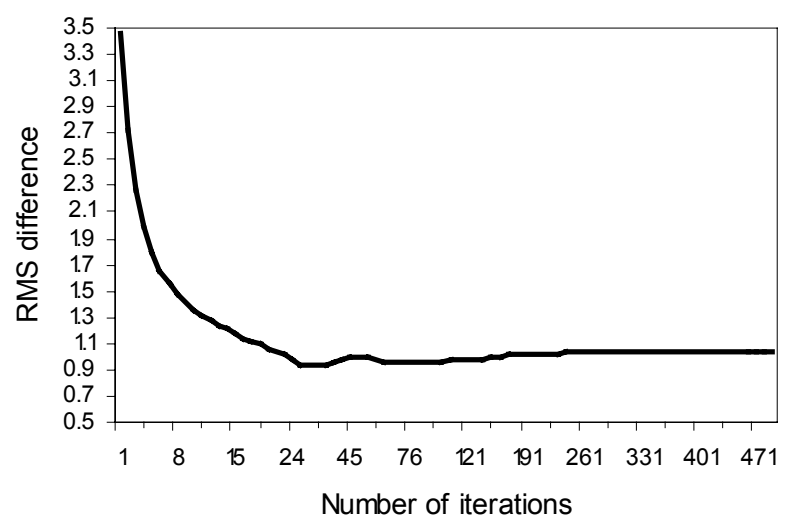

"red" experiment
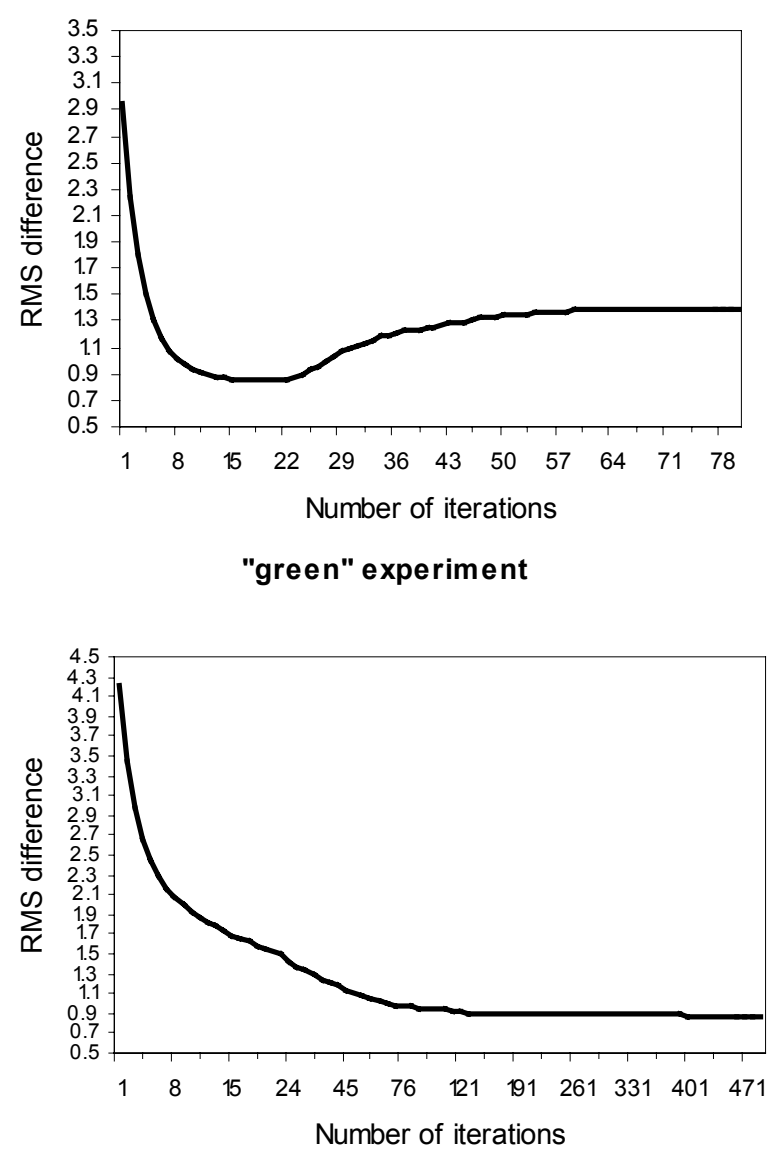

"yellow" experiment

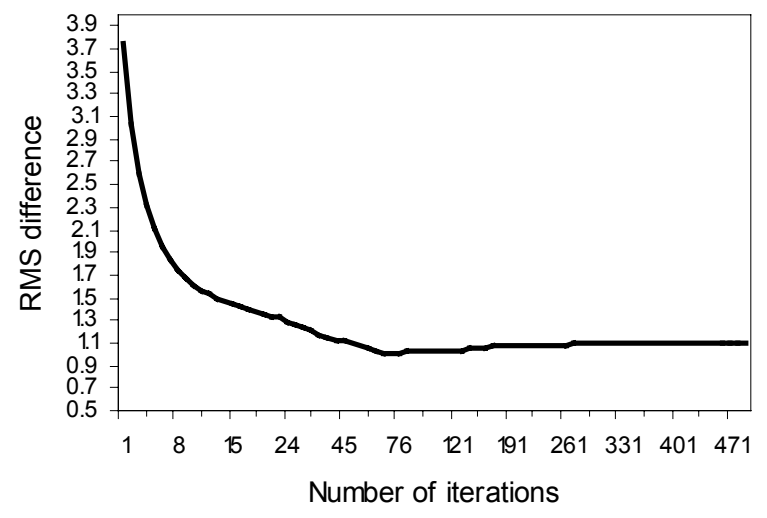

Figure 1: The accuracy of retinex as a model as measured by the RMS difference between the retinex output and the MMT corresponding color data as a function of the number of retinex parameter nIterations ${ }^{2}$. 


\section{CONCLUSIONS}

For the MMT experiments we have been able to solve for the parameters needed in the retinex algorithm, based on a best fit with the experimental data. We have found that if we run either of the Matlab retinex algorithms ${ }^{2}$, the optimum results can be obtained by choosing the number of iterations to be 33. An interesting finding was that the image resolution has very little effect on the accuracy of prediction. Details of the post-retinex processing step were also established. Although we have established parameters for the retinex computation based on the available MMT data, it would be helpful to have more extensive experimental data to improve the reliability of the results.

\section{ACKNOWLEDGMENTS}

We are grateful for the financial support of the Natural Sciences and Engineering Research Council of Canada.

\section{REFERENCES}

1. Frankle, J., and McCann, J.J., "Method and Apparatus for Lightness Imaging”, US Patent \#4,384,336, May 17, 1983.

2. Funt, B.V., Ciurea, F., and McCann, J.J., "Retinex in Matlab" Proc. IS\&T/SID Eighth Color Imaging Conference, 112-121, Scottsdale 2000.

3. Glasser, L.G., McKinney, A.H., Reilly, C.D., and Schnelle, P.D. "Cube-root color coordinate system”. J. Opt. Soc. Am. 48, 736-740, 1958.

4. Hunt, R.W.G., "Measuring Colour” ( $3^{\text {rd }}$ edition), Fountain Press, p. 210,1998.

5. Land, E., "The Retinex Theory of Color Vision", Scientific American, 237, 108 - 128, 1977.

6. Land, E., "An alternative technique for the computation of the designator in the retinex theory of color vision", Proc. of the National Academy of Science USA, 83, 2078-3080, May 1986.

7. Land, E. and McCann, J.J., "Lightness and Retinex Theory", Journal of the Optical Society of America, 61(1), January 1971.

8. McCann, J.J., "Lessons Learned from Mondrians Applied to Real Images and Color Gamuts", Proc. IS\&T/SID Seventh Color Imaging Conference, pp. 1-8, 1999.

9. McCann, J.J., McKee, S.P., and Taylor, T.H., "Quantitative Studies in Retinex Theory: A Comparison Between Theoretical Predictions and Observer Responses to the 'Color Mondrian' Experiments", J. of Vision Res. Vol. 16, $445-458,1976$.

10. Nayatani, Y., Takahama, K., Sobagaki, H., "Prediction of Color Appearance of Object Colors in a Complex Visual Field", J. Light \& Vis. Env. Vol. 19, No. 1, 1995.

\footnotetext{
* Correspondence: funt@sfu.ca; phone 604-291-3126; fax 604-291-3045; http://css.sfu.ca/members/funt.html; School of Computing Science, Simon Fraser University, 8888 University Drive, Burnaby, B.C. Canada V5A 1S6
} 